Mathematical Modelling and Analysis

Volume 22 Number 3, May 2017, 337-351

https://doi.org/10.3846/13926292.2017.1309702

(c) Vilnius Gediminas Technical University, 2017
Publisher: Taylor\&Francis and VGTU

http://www.tandfonline.com/TMMA

ISSN: $1392-6292$

eISSN: 1648-3510

\title{
Uniformly Valid Asymptotics for Carrier's Mathematical Model of String Oscillations
}

\author{
Paulius Miškinis ${ }^{a}$, Aleksandras $\operatorname{Krylovas}^{a}$ and \\ Olga Lavcel-Budko ${ }^{b}$
}

${ }^{a}$ Vilnius Gediminas Technical University

Saulètekio al. 11, LT-10223 Vilnius, Lithuania

${ }^{b}$ Mykolas Romeris University

Ateities st. 20, LT-08303 Vilnius, Lithuania

E-mail(corresp.): akr@fm.vgtu.1t

E-mail: paulius.miskinis@vgtu.lt

Received October 4, 2016; revised January 31, 2017; published online May 15, 2017

\begin{abstract}
In the paper, an asymptotic analysis of G.F. Carrier's mathematical model of string oscillation is presented. The model consists of a system of two nonlinear second order partial differential equations and periodic initial conditions. The longitudinal and transversal string oscillations are analyzed together when at the initial moment of time the system's solutions have amplitudes proportional to a small parameter. The problem is reduced to a system of two weakly nonlinear wave equations. The resonant interaction of periodic waves is analyzed. An uniformly valid asymptotic approximation in the long time interval, which is inversely proportional to the small parameter, is constructed. This asymptotic approximation is a solution of averaged along characteristics integro-differential system. Conditions of appearance of combinatoric resonances in the system have been established. The results of numerical experiments are presented.
\end{abstract}

Keywords: asymptotic analysis, averaging, nonlinear waves, resonances.

AMS Subject Classification: 35L60; 41A60; 74J30.

\section{The mathematical model}

The linear string oscillation equation is

$$
v_{t t}-b^{2} v_{x x}=0
$$

here $x \in[0, l]$ is a coordinate, $t \in[0, \infty)$ - time, $b>0$ - sound velocity, and $v(x, t)$ is the string transverse deviation from the equilibrium position which, together with the initial and boundary conditions, makes the well-known smallamplitude string fluctuation model (see, e. g., [30]). 
Equation (1.1) is obtained by introducing certain mathematical and physical preconditions limiting the applicability of the model. For example, mathematical preconditions regarding the smallness of the function $v(x, t):|v(x, t)| \ll 1$, analyticity or at least smoothing $v(x, t) \in C^{2}([0, l] \times[0, \infty))$, physical preconditions regarding string weightlessness, nontensility and absolute tensility. In the opposite case, we should account for the effect of gravitational forces and for the fact that the string's length in the process of its motions may change, therefore the Hooke tensility law does not work.

If we reject at least one above-mentioned or other "obvious" precondition, we shall obtain various generalizations of the linear wave equation (1.1) and of the related model.

For example, upon rejecting the conditions of the small deviation and given that $v(x, t) \sim 1$, the equation of motion begins to depend on the first-order derivative $v_{x}(x, t)$ and becomes nonlinear. We can show that in this case from the Lagrangian function, corresponding to such an oscillating string

$$
\mathcal{L}=\frac{1}{2} \rho A v_{t}^{2}-T\left(\sqrt{1+v_{x}^{2}}-1\right)
$$

with the usual boundary conditions $v(0, t)=v(l, t)=0$, there follows a more complicated equation of motion

$$
v_{t t}=\frac{b^{2} v_{x x}}{\left(1+v_{x}^{2}\right)^{3 / 2}}, \quad b=\frac{T}{\rho A} .
$$

Employing mechanical reasonings, this equation was obtained [22].

A broader context of modelling is presented in the monographs $[12,31]$. The monograph [31] deals with the general properties of the mathematical modelling whereas the monograph [12] helps us to find asymptotic solutions of the strongly nonlinear systems of differential equations. The monograph is dedicated to a very important fundamental problem: the inversion of Lagranges theorem on the stability of equilibrium not a trivial task, over which researchers had struggled for more than half a century.

The oscillation equations of the both mentioned models have their regions of validity. Equation (1.2) is nonlinear, valid also in the case when the value of the transversal deviations $|v(x, t)| \gg 1$. However, in the case transversal deviations are too large, equation (1.2) becomes misleading: in case of large deviations, the deflection of a string element may be not only transversal, but also longitudinal. This means that large deviations are characterized by two functions: transversal $v(x, t)$ and longitudinal $u(x, t)$. It is the idea that has stimulated a number of authors to formulate the corresponding nonlinear models of a fluctuating string not for one but for two functions $-v(x, t)$ and $u(x, t)$ (see, e. g., $[1,2,7,21])$.

For example, this attracted G.F.Carrier's attention in $[5,6]$ (see also $[8,25]$ ); he has formulated a model of the nonlinear motion of a string, in which he analyzes both transversal and longitudinal string oscillations, describing them 
by a system of two nonlinear second-order partial differential equations:

$$
\left\{\begin{array}{l}
\rho A u_{t t}-E A u_{x x}-(E A-T) \frac{\left(1+u_{x}\right) v_{x} v_{x x}-u_{x x} v_{x}^{2}}{\sqrt{\left[\left(1+u_{x}\right)^{2}+v_{x}^{2}\right]^{3}}}=0, \\
\rho A v_{t t}-E A v_{x x}+(E A-T) \frac{\left(1+u_{x}\right)^{2} v_{x x}-\left(1+u_{x}\right) u_{x x} v_{x}}{\sqrt{\left[\left(1+u_{x}\right)^{2}+v_{x}^{2}\right]^{3}}}=0,
\end{array}\right.
$$

here $\rho$ is the substance density, $A$ - the transection area, $E$ - the Young modulus of string, $T$ - the initial tension. Differently from the classical rectilinear motion, an element of a non-stimulated string is characterized by the spatial axial coordinate $x$ and the time moment $t$. Deviations of the stimulated string element are characterized by the longitudinal $u(x, t)$ and transverse $v(x, t)$ displacements. The string moves in the $(u, v)$ plane. Such a note may be regarded as a parametrical curve which depends not only on the spatial but also on the time coordinate for which the radius-vector $r=(u(x, t), v(x, t))$.

What is the interrelation of the equations of motion (1.1), (1.2) and (1.3)? In the absence of longitudinal oscillations, i.e. when $u(x, t) \equiv u_{x}(x, t) \equiv$ $u_{x x}(x, t) \equiv 0$, system (1.3) degenerates into one equation which characterizes only transverse oscillations $v(x, t)$ :

$$
\rho A v_{t t}-\frac{T v_{x x}}{\left(1+v_{x}^{2}\right)^{3 / 2}}-E A\left[v_{x x}-\frac{v_{x x}}{\left(1+v_{x}^{2}\right)^{3 / 2}}\right]=0 .
$$

In case when the condition

$$
v_{x}^{2} \ll \frac{2}{3} \frac{T}{E A}
$$

is valid, the last term in equation (1.4) may be ignored, and this equation transforms into equation (1.2). Indeed, when $\left|v_{x}^{2}\right| \ll 1$, using the series

$$
\left(1+v_{x}^{2}\right)^{3 / 2}=1+\frac{3}{2} v_{x}^{2}+O\left(v_{x}^{4}\right)
$$

equation (1.4) may be written as follows:

$$
\rho A v_{t t}-\frac{T v_{x x}}{\left(1+v_{x}^{2}\right)^{3 / 2}}-\frac{\frac{3}{2} E A v_{x x} v_{x}^{2}}{\left(1+v_{x}^{2}\right)^{3 / 2}}=0 .
$$

The second term will be much larger than the third one if its numerator will be significantly larger than the third term numerator, i.e.

$$
T \gg \frac{3}{2} E A v_{x}^{2},
$$

and it is from here that the condition (1.5) follows.

It is well known (see, e. g., [4]) that $\frac{T}{E A}$ is a deformation or, in our case, the relative lengthening of the string $\frac{\Delta l}{l}$. Condition (1.5) has a simple geometrical interpretation:

$$
v_{x}^{2} \ll \frac{2}{3} \frac{\Delta l}{l} .
$$


Inequality (1.8) and expansion (1.6) will be satisfied if $\Delta l<\frac{3}{2} l$.

Let us summarize: when a swaying string lengthens more than $3 / 2$ times, the Carrier's model (1.3) should be used. When the lengthening does not exceed more than $3 / 2$ of the string length but is rather large, e.g., $\Delta l \sim l$, model (1.2) may be applied. Last but not least, when the condition $\Delta l \ll l$ and correspondingly $v_{x}^{2} \ll 1$, equation (1.2) turns into the classical rectilinear swaying equation (1.1). Note that the presented limitations of the application of the models are valid in the bounded region of changing the independent variables $(x, t) \in[0, l] \times\left[0, t^{0}\right]$, when the constants $l, t^{0}$ do not depend on the asymptotic relations (1.5), (1.7), etc. The more subtle asymptotic analysis performed in this paper shows that when a large $(x, t)$ region of variable changes is analyzed, transition to a simpler model may be groundless even at small oscillation amplitudes.

Coming back to system (1.3), let us note that when at the initial moment of time $t=0$ the system solutions have small amplitudes (proportional to the small parameter $\varepsilon$ ), the problem is reduced to a system of two nonlinear wave equations. In this paper, we construct an asymptotic approximation uniformly valid in the long time interval $t \sim \varepsilon^{-1}$ and determine the appearance conditions of the resonant wave interactions.

\section{Analysis of the model by the small parameter method}

Suppose that at the initial moment of time $t=0$ the following conditions are satisfied:

$$
\begin{aligned}
& u(x, 0)=\tilde{\varepsilon} u_{0}(x), u_{t}(x, 0)=\tilde{\varepsilon} u_{1}(x), \\
& v(x, 0)=\tilde{\varepsilon} v_{0}(x), v_{t}(x, 0)=\tilde{\varepsilon} v_{1}(x), 0<\tilde{\varepsilon} \ll 1 .
\end{aligned}
$$

We shall search for the system (1.3) solution in the form $u=\tilde{\varepsilon} \tilde{u}, v=\tilde{\varepsilon} \tilde{v}$. Substituting $u, v$ in (1.3) and selecting the terms of $\tilde{\varepsilon}$ power-series expansions, we obtain:

$$
\begin{aligned}
& \tilde{\varepsilon}\left(\rho A \tilde{u}_{t t}-E A \tilde{u}_{x x}\right)-\tilde{\varepsilon}^{2}(E A-T) \\
& \quad \times\left(\tilde{v}_{x} \tilde{v}_{x x}+\tilde{\varepsilon}\left(\tilde{u}_{x x} \tilde{v}_{x}^{2}-2 \tilde{u}_{x} \tilde{v}_{x} \tilde{v}_{x x}+O(\tilde{\varepsilon})\right)\right)=0, \\
& \tilde{\varepsilon}\left(\rho A \tilde{v}_{t t}-T \tilde{v}_{x x}\right)-\tilde{\varepsilon}^{2}(E A-T)\left(\tilde{u}_{x x} \tilde{v}_{x}+\tilde{u}_{x} \tilde{v}_{x x}\right. \\
& \left.\quad-\tilde{\varepsilon}\left(\tilde{u}_{x}^{2} \tilde{v}_{x x}-\frac{3}{2} \tilde{v}_{x x} \tilde{v}_{x}^{2}+2 \tilde{u}_{x} \tilde{v}_{x} \tilde{u}_{x x}+O(\tilde{\varepsilon})\right)\right)=0 .
\end{aligned}
$$

Let us note that

$$
a^{2}=E / \rho, \quad b^{2}=T /(\rho A), \quad \varepsilon=\tilde{\varepsilon}\left(b^{2}-a^{2}\right) .
$$

By omitting members of the $O\left(\tilde{\varepsilon}^{3}\right)$ order and neglecting the sign ( $\left.{ }^{\sim}\right)$, we receive the asymptotic integration problem

$$
\left\{\begin{array}{l}
u_{t t}-a^{2} u_{x x}=-\varepsilon v_{x} v_{x x} \\
v_{t t}-b^{2} v_{x x}=-\varepsilon\left(v_{x} u_{x x}+u_{x} v_{x x}\right)
\end{array}\right.
$$


with the initial conditions corresponding to (2.1). When in system $(2.3) \varepsilon=0$, we obtain a couple of two independent travelling waves (see, e. g., [30]):

$$
\begin{aligned}
& u(x, t)=\frac{1}{2}\left(u_{0}(x+a t)+u_{0}(x-a t)\right)+\frac{1}{2 a} \int_{x-a t}^{x+a t} u_{1}(\xi) d \xi \\
& v(x, t)=\frac{1}{2}\left(v_{0}(x+b t)+v_{0}(x-b t)\right)+\frac{1}{2 b} \int_{x-b t}^{x+b t} v_{1}(\xi) d \xi .
\end{aligned}
$$

Functions (2.4) are obtained also from the linear model (1.1). However, when $\varepsilon>0$, function (2.4) will be close to the solution of the system (2.3), in the general case only in a short time interval $t \ll O\left(\varepsilon^{-1}\right)$.

The system (2.1)-(2.3) has a classical (continuously differentiable with respect to the variables $t$ ir $x)$ solution $u(t, x ; \varepsilon), v(t, x ; \varepsilon)$ in the time interval $t \in\left[0, \frac{\tau^{0}}{\varepsilon}\right]$, here $\tau^{0}$ is a constant. We are constructing an asymptotic solution $U(t, x ; \varepsilon), V(t, x ; \varepsilon)$ uniformly valid in the indicated interval: $\forall \mu>0 \exists \varepsilon_{\mu}>0$ such, that for all $\varepsilon \in\left(0, \varepsilon_{\mu}\right]$ is true next estimation

$$
\max _{t \in\left[0, \frac{\tau^{0}}{\varepsilon}\right], x \in R}|U(t, x ; \varepsilon)-u(t, x ; \varepsilon)|+|V(t, x ; \varepsilon)-v(t, x ; \varepsilon)|<\mu .
$$

At a certain moment of time $t=\frac{\tau^{1}}{\varepsilon}$, there may appear the discontinuous derivatives $u_{t}, u_{x}, v_{t}, v_{x}$ (gradient catastrophe) is constructed and its asymptotic approximation does not describe the solution. This means that the time interval under analysis $t \in\left[0, \frac{\tau^{0}}{\varepsilon}\right]$ and $\tau^{0}<\tau^{1}$. Estimation (2.5) is an analog of the well known in asymptotic analysis N.N. Bogoliubov's theorem [24].

The aspects of mathematical substantiation of asymptotic approximations constructed in the paper have been analyzed $[13,14]$.

\section{Construction of the uniformly valid asymptotics}

Let us rewrite system (2.3) in the Riemann invariants $r_{j}$ of the linear part of the system. Note that the asymptotic method does not use the quasi-linear system invariants which may be constructed by the methods of [9] [10].

$$
r_{1}=u_{t}-a u_{x}, \quad r_{2}=u_{t}+a u_{x}, r_{3}=v_{t}-b v_{x}, r_{4}=v_{t}+b v_{x} .
$$

Then we obtain

$$
\left\{\begin{aligned}
\frac{\partial r_{j}}{\partial t} \pm a \frac{\partial r_{j}}{\partial x}=- & \frac{\varepsilon}{4 b^{2}}\left(r_{4}-r_{3}\right)\left(r_{4 x}-r_{3 x}\right), \quad j=1,2 \\
\frac{\partial r_{j}}{\partial t} \pm b \frac{\partial r_{j}}{\partial x}=- & \frac{\varepsilon}{4 a b}\left(\left(r_{4}-r_{3}\right)\left(r_{2 x}-r_{1 x}\right)\right. \\
& \left.+\left(r_{2}-r_{1}\right)\left(r_{4 x}-r_{3 x}\right)\right), \quad j=3,4
\end{aligned}\right.
$$

We shall analyze system (3.1) when functions (2.1) are $2 \pi$-periodic:

$$
r_{j}(0, x ; \varepsilon)=\sum_{k_{j}=-\infty}^{+\infty} r_{j k_{j}}^{(0)} e^{\mathrm{i} k_{j} x} .
$$


If to looking for the asymptotic solution of (3.1) as a regular small parameter $\varepsilon$ power series expansion

$$
r_{j}(t, x ; \varepsilon)=r_{j 0}(t, x)+\varepsilon r_{j 1}(t, x)+O\left(\varepsilon^{2}\right),
$$

we shall see that in the general case (3.3) we will have the secular terms $\varepsilon t$ (see, e. g., [26]). Therefore, approximation (3.3) may be used only when the values $\varepsilon t$ are small, i. e. $t \ll \varepsilon^{-1}$, or in a short time $t$ interval. On the other hand, equations (3.1) have a classical (diferentiated) solution when $t \in\left[0, O\left(\varepsilon^{-1}\right)\right]$, i. e. in a long time interval.

Let us note that in papers $[5,6]$ concerning the model (1.3) the author analyzed the asymptotics, but they had secular terms.

It is a non-trivial problem to construct an asymptotic solution uniformly valid in a long time interval $t \in\left[0, O\left(\varepsilon^{-1}\right)\right]$. Such asymptotics

$$
r_{j}(x, t ; \varepsilon)=R_{j}\left(\tau, y_{j}\right)+O(\varepsilon)
$$

is found as a solution of the following system averaged along the characteristics $[15,16]$ :

$$
\left\{\begin{aligned}
\frac{\partial R_{j}}{\partial \tau}=-\frac{1}{4 b^{2}}\langle & \left(R_{4}\left(\tau, y_{4}\right)-R_{3}\left(\tau, y_{3}\right)\right) \\
& \left.\left(\frac{\partial R_{4}\left(\tau, y_{4}\right)}{\partial y_{4}}-\frac{\partial R_{3}\left(\tau, y_{3}\right)}{\partial y_{3}}\right)\right\rangle_{j}, j=1,2, \\
\frac{\partial R_{3}}{\partial \tau}=-\frac{1}{4 a b}\langle & \left(R_{4}\left(\tau, y_{4}\right)\left(\frac{\partial R_{2}\left(\tau, y_{2}\right)}{\partial y_{2}}-\frac{\partial R_{1}\left(\tau, y_{1}\right)}{\partial y_{1}}\right)+\right. \\
& \left.\left.\left(\left(R_{2}\left(\tau, y_{2}\right)-R_{1}\left(\tau, y_{1}\right)\right) \frac{\partial R_{4}\left(\tau, y_{4}\right)}{\partial y_{4}}\right)\right)\right\rangle_{3}, \\
\frac{\partial R_{4}}{\partial \tau}=\frac{1}{4 a b}\langle & \left(R_{3}\left(\tau, y_{3}\right)\left(\frac{\partial R_{2}\left(\tau, y_{2}\right)}{\partial y_{2}}-\frac{\partial R_{1}\left(\tau, y_{1}\right)}{\partial y_{1}}\right)\right\rangle_{+} \\
& \left.\left.\left(\left(R_{2}\left(\tau, y_{2}\right)-R_{1}\left(\tau, y_{1}\right)\right) \frac{\partial R_{3}\left(\tau, y_{3}\right)}{\partial y_{3}}\right)\right)\right\rangle_{4}
\end{aligned}\right.
$$

Here, $\tau=\varepsilon t$ is a slow time, $y_{1}=x-a t, y_{2}=x+a t, y_{3}=x-b t, y_{4}=x+b t$ are the fast characteristic variables, $y=\left(y_{1}, y_{2}, y_{3}, y_{4}\right)$. The operators $\langle\cdots\rangle_{j}$ averaging along characteristics are described as follows:

$$
\begin{aligned}
& \langle f(\tau, y)\rangle_{1}= \\
& \lim _{T \rightarrow \infty} \frac{1}{T} \int_{0}^{T} f\left(\tau, y_{1}, y_{1}+\left(\lambda_{1}-\lambda_{2}\right) s, y_{1}+\left(\lambda_{1}-\lambda_{3}\right) s, y_{1}+\left(\lambda_{1}-\lambda_{4}\right) s\right) d s, \\
& \langle f(\tau, y)\rangle_{2}= \\
& \lim _{T \rightarrow \infty} \frac{1}{T} \int_{0}^{T} f\left(\tau, y_{2}+\left(\lambda_{2}-\lambda_{1}\right) s, y_{2}, y_{2}+\left(\lambda_{2}-\lambda_{3}\right) s, y_{2}+\left(\lambda_{2}-\lambda_{4}\right) s\right) d s, \\
& \langle f(\tau, y)\rangle_{3}= \\
& \lim _{T \rightarrow \infty} \frac{1}{T} \int_{0}^{T} f\left(\tau, y_{3}+\left(\lambda_{3}-\lambda_{1}\right) s, y_{3}+\left(\lambda_{3}-\lambda_{2}\right) s, y_{3}, y_{3}+\left(\lambda_{3}-\lambda_{4}\right) s\right) d s, \\
& \langle f(\tau, y)\rangle_{4}= \\
& \lim _{T \rightarrow \infty} \frac{1}{T} \int_{0}^{T} f\left(\tau, y_{4}+\left(\lambda_{4}-\lambda_{1}\right) s, y_{4}+\left(\lambda_{4}-\lambda_{2}\right) s, y_{4}+\left(\lambda_{4}-\lambda_{3}\right) s, y_{4}\right) d s .
\end{aligned}
$$

Here, $\lambda_{1}=a, \lambda_{2}=-a, \lambda_{3}=b, \lambda_{4}=-b$. 
When all numbers $\frac{\lambda_{i}-\lambda_{j}}{\lambda_{k}-\lambda_{j}}$ are irrational, the right sides of system (3.4) will be equal to zero and the solutions are expressed by formulas (2.4), i. e. travelling by independent waves at the velocity $\lambda_{j}$. In the opposite case, the right sides of system (3.4) may not equal zero, and in this case the solution of this system depends on the slow time $\tau$. In this paper, we shall limit ourselves to the case when $a$ and $b$ are integer numbers.

We shall obtain an integral differential system which we integrate from 0 to $2 \pi$. Sometimes such averaging is called internal [15], and this is its essential difference as compared with various partial derivatives averaging schemes $[23,24]$ which may be called the external averaging. The systems obtained by internal averaging often remain without a subsequent study and left in the literature as a certain theoretical result of the asymptotic analysis [3,28]. Let us note that system (3.4) does not directly depend on the small parameter $\varepsilon$ and thus has no problems of asymptotic integration. Therefore, the problem may be successfully solved by numerical methods $[15,16]$. Let us note some papers $[11,20,27,29]$ in which, like in our work, the method of two scales and averaging methods are applied, however, the Fourier analysis is performed without directly constructing the averaging system. In our paper, for the definite equations (1.3), we have applied the general method of averaging along characteristics the weakly nonlinear hyperbolic systems. The constructed averaging system allows finding uniformly valid asymptotic approximations of a polynomial form by applying the methods of our earlier work [17]. On the other hand, the theoretical analysis of the obtained averaged system (see $[18,19]$ ) allows determining the conditions of appearing combinatoric resonances.

\section{Analysis of the averaged system}

We shall analyze the solution of system (3.4) by the Fourier series:

$$
R_{j}\left(\tau, y_{j}\right)=\sum_{k_{j}=-\infty}^{+\infty} R_{j k_{j}}(\tau) e^{\mathbf{i} k_{j} y_{j}} .
$$

The functions $R_{j k_{j}}(\tau)$ (3.2) satisfy the initial conditions $R_{j k_{j}}(0)=r_{j k_{j}}^{(0)}$. When the right sides of system (3.4) are equal to zero, correspondingly the functions $R_{j k_{j}}(\tau) \equiv r_{j k_{j}}^{(0)}$, i. e. do not depend on $\tau$. Otherwise we shall have the resonant wave interaction and the functions $R_{j k_{j}}(\tau)$ will describe the slowly changing wave amplitude.

Let us denote the sets of resonant harmonics:

$$
\mathcal{R}_{j}=\left\{k_{j} \in Z \backslash\{0\}: \frac{d R_{j k_{j}}(\tau)}{d \tau} \not \equiv 0\right\} .
$$

The resonant wave interaction in the system does not appear if $\mathcal{R}_{1}=\mathcal{R}_{2}=$ $\mathcal{R}_{3}=\mathcal{R}_{4}=\emptyset$. The sets $\mathcal{R}_{j}$ are interdependent and are empty only in case when the parameters of the problem $a=\sqrt{\frac{E}{\rho}}$ and $b=\sqrt{\frac{T}{\rho A}}$ meet certain requirements. Suppose that the sets $\mathcal{R}_{3}$ and $\mathcal{R}_{4}$ have no such harmonics $k_{3}, k_{4}$ 
for whom

$$
k_{3}(a \mp b)=k_{4}(a \pm b)
$$

is valid. In this case, we shall have $\mathcal{R}_{1}=\mathcal{R}_{2}=\emptyset$. On the other hand, the sets $\mathcal{R}_{3,4}$ will be empty, if they and the sets $\mathcal{R}_{1,2}$ have no such harmonics for which

$$
k_{1,2}(2 b)=k_{3,4}(a \pm b)
$$

is valid.

Note also that for resonances to appear the following relations among all harmonics depending to the set $\mathcal{R}_{j}$ are necessary:

$$
k_{1,2}=k_{3}+k_{4}, k_{3,4}=k_{4,3}+k_{1,2} .
$$

Let us note that the relations (4.3), (4.4) imply that the corresponding coefficients of the Fourier $R_{j k_{j}}(\tau)$ should not be equal to zero.

\section{Numerical experiments}

In practice, we limit ourselves to the first Fourier lines (4.1) harmonics $\left|k_{j}\right| \leq K$ and look for the functions $R_{j k_{j}}(\tau)$ by the following approximations:

$$
R_{j k_{j}}(\tau) \approx R_{j k_{j}}^{(0)}+R_{j k_{j}}^{(1)} \tau+R_{j k_{j}}^{(2)} \tau^{2}+\cdots+R_{j k_{j}}^{(N)} \tau^{N}
$$

We find the polynomial coefficients by the method of undetermined coefficients. The same results we obtain by successive iterations.

\subsection{Example 1}

We shall analyze the problem when $a=1, b=3$ and the initial conditions are as follows:

$$
\begin{aligned}
& R_{1}\left(0, y_{1}\right)=R_{2}\left(0, y_{2}\right)=0 \\
& R_{3}\left(0, y_{3}\right)=\sin 2 y_{3}+\sin 4 y_{3}+2 \cos 2 y_{3}+\cos 4 y_{3} \\
& R_{4}\left(0, y_{4}\right)=3 \sin 2 y_{4}+\sin 4 y_{4}+5 \cos 2 y_{4}+\cos 4 y_{4}
\end{aligned}
$$

We shall present the calculated data using the computer algebra system Maple coefficients (5.1):

$$
\begin{array}{r}
R_{1}\left(\tau, y_{1}\right)=\left(\frac{2}{3} \tau-\frac{11}{216} \tau^{3}+\frac{227}{311040} \tau^{5}+\frac{12463}{313528320} \tau^{7}\right) \cos 6 y_{1} \\
+\left(-\frac{1}{6} \tau+\frac{11}{864} \tau^{3}-\frac{227}{1244160} \tau^{5}+\frac{12463}{1254113280} \tau^{7}\right) \sin 6 y_{1} \\
R_{2}\left(\tau, y_{2}\right)=\left(\frac{1}{4} \tau-\frac{1}{432} \tau^{3}-\frac{11}{622080} \tau^{5}+\frac{23}{67184640} \tau^{7}\right) \cos 6 y_{2} \\
+\left(-\frac{1}{12} \tau+\frac{1}{1296} \tau^{3}+\frac{11}{1866240} \tau^{5}-\frac{23}{201553920} \tau^{7}\right) \sin 6 y_{2}
\end{array}
$$




$$
\begin{aligned}
R_{3}( & \left., y_{3}\right)=\left(2+\frac{1}{72} \tau^{2}-\frac{19}{62208} \tau^{4}+\frac{181}{134369280} \tau^{6}\right) \cos 2 y_{3} \\
+ & \left(1+\frac{1}{144} \tau^{2}-\frac{19}{124416} \tau^{4}+\frac{181}{268738560} \tau^{6}\right) \sin 2 y_{3} \\
& +\left(1-\frac{17}{72} \tau^{2}+\frac{85}{10368} \tau^{4}+\frac{17}{414720} \tau^{6}\right) \cos 4 y_{3} \\
& +\left(1-\frac{17}{72} \tau^{2}+\frac{85}{10368} \tau^{4}+\frac{17}{414720} \tau^{6}\right) \sin 4 y_{3}, \\
R_{4}( & \left., y_{4}\right)=\left(5+\frac{5}{144} \tau^{2}-\frac{25}{4608} \tau^{4}+\frac{1889}{5971968} \tau^{6}\right) \cos 2 y_{4} \\
& +\left(3+\frac{1}{48} \tau^{2}-\frac{5}{1536} \tau^{4}+\frac{1889}{9953280} \tau^{6}\right) \sin 2 y_{4} \\
& +\left(1-\frac{5}{144} \tau^{2}+\frac{5}{124416} \tau^{4}+\frac{179}{53747712} \tau^{6}\right) \cos 4 y_{4} \\
& +\left(1-\frac{5}{144} \tau^{2}+\frac{5}{124416} \tau^{4}+\frac{179}{53747712} \tau^{6}\right) \sin 4 y_{4} .
\end{aligned}
$$

We see that the sets $\mathcal{R}_{1,2}=\{-6,6\}, \mathcal{R}_{3,4}=\{-4,-2,2,4\}$. Thus, conditions (4.2), (4.3) are satisfied: $2 k_{3}=4 k_{4}, 6 k_{3,4}=2 k_{1,2}$ or $6 k_{3,4}=4 k_{1,2}$. Besides, all harmonics satisfy conditions (4.4): $\pm 6= \pm 4 \pm 2, \pm 4= \pm 6 \mp 2$ and $\pm 2=\mp 4 \pm 6$.

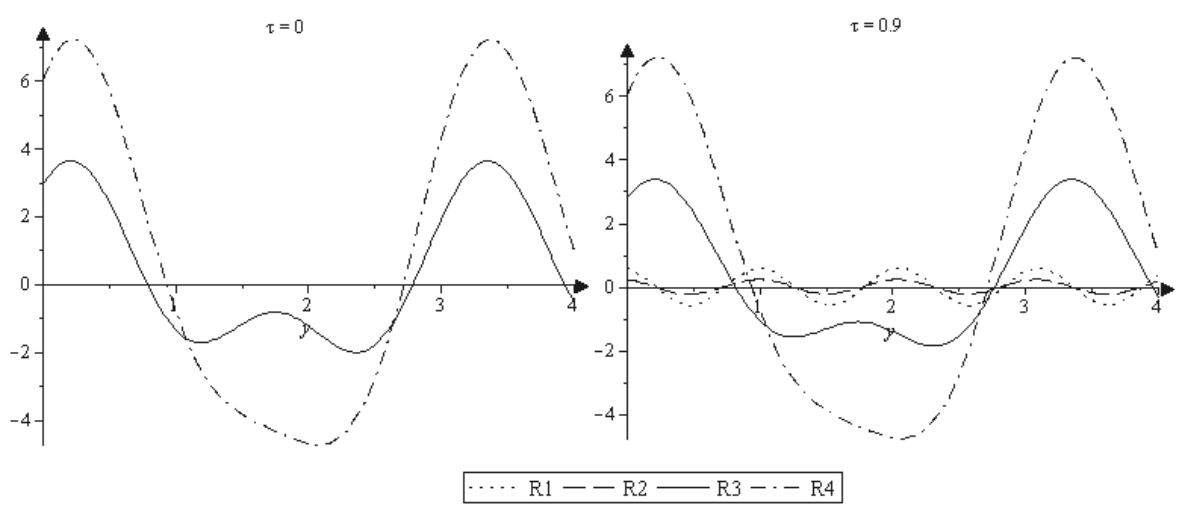

Figure 1. Graphs of example 1 functions $R_{1}, R_{2}, R_{3}, R_{4}$

Figure 1 presents graphs of the functions $R_{j}(\tau, y), j=1,2,3,4$ when $\tau=0$ and $\tau=0.9$, and $y_{1}=y_{2}=y_{3}=y_{4}=y$ are taken. At the initial time moment $\tau=0$, the functions $R_{1}$ and $R_{2}$ are absent, and at $\tau=0.9$ there appear waves, their amplitudes depending on $\tau$. The resonant interactions of waves cause also the dependence of the amplitudes of waves $R_{3}, R_{4}$ on the slow time $\tau$. The amplitudes slowly change, however, at $\tau=0.9$ in the graph the new waves $R_{3}$, $R_{4}$ are clearly seen. 


\subsection{Example 2}

Let us take the same values as in Fig. 1: $a=1, b=3$, and in the initial conditions let us take the non-zero functions $R_{1}, R_{2}$ :

$$
\begin{aligned}
& R_{1}\left(0, y_{1}\right)=\sin 3 y_{1}+5 \cos 3 y_{1}, \\
& R_{2}\left(0, y_{2}\right)=4 \sin 3 y_{2}+8 \cos 3 y_{2}, \\
& R_{3}\left(0, y_{3}\right)=\sin 2 y_{3}+\sin 4 y_{3}+2 \cos 2 y_{3}+\cos 4 y_{3}, \\
& R_{4}\left(0, y_{4}\right)=3 \sin 2 y_{4}+\sin 4 y_{4}+5 \cos 2 y_{4}+\cos 4 y_{4} .
\end{aligned}
$$

\begin{tabular}{|c|c|c|c|c|c|c|c|}
\hline & & $\tau^{0}$ & $\tau^{1}$ & $\tau^{2}$ & $\tau^{3}$ & $\tau^{4}$ & $\tau^{5}$ \\
\hline \multirow{4}{*}{$R_{1}$} & $\cos 3 y_{1}$ & 5 & & 0.0217 & & -0.0005 & \\
\hline & $\sin 3 y_{1}$ & 1 & & 0.0043 & & -0.0001 & \\
\hline & $\cos 6 y_{1}$ & & 0.6667 & & -0.0818 & & 0.0080 \\
\hline & $\sin 6 y_{1}$ & & -0.1667 & & 0.0204 & & -0.0020 \\
\hline \multirow{4}{*}{$R_{2}$} & $\cos 3 y_{2}$ & 8 & & 0.2361 & & -0.0196 & \\
\hline & $\sin 3 y_{2}$ & 4 & & 0.1181 & & -0.0098 & \\
\hline & $\cos 6 y_{2}$ & & 0.25 & & -0.0061 & & 0.0001 \\
\hline & $\sin 6 y_{2}$ & & -0.0833 & & 0.0020 & & $0.394 \cdot 10^{-6}$ \\
\hline \multirow{6}{*}{$R_{3}$} & $\cos y_{3}$ & & 0.1667 & & -0.0057 & & -0.0002 \\
\hline & $\sin y_{3}$ & & 2.1667 & & -0.0740 & & -0.0032 \\
\hline & $\cos 2 y_{3}$ & 2 & & 0.0764 & & -0.0001 & \\
\hline & $\sin 2 y_{3}$ & 1 & & -0.0382 & & $-4.823 \cdot 10^{-6}$ & \\
\hline & $\cos 4 y_{3}$ & 1 & & -0.2361 & & 0.0301 & \\
\hline & $\sin 4 y_{3}$ & 1 & & -0.2361 & & 0.0301 & \\
\hline \multirow{6}{*}{$R_{4}$} & $\cos y_{4}$ & & 0.1250 & & -0.0014 & & $-0.803 \cdot 10^{-7}$ \\
\hline & $\sin y_{4}$ & & 0.4583 & & -0.0052 & & $-0.294 \cdot 10^{-6}$ \\
\hline & $\cos 2 y_{4}$ & 5 & & -0.6597 & & -0.0046 & \\
\hline & $\sin 2 y_{4}$ & 3 & & -0.3958 & & -0.0028 & \\
\hline & $\cos 4 y_{4}$ & 1 & & -0.0347 & & 0.0011 & \\
\hline & $\sin 4 y_{4}$ & 1 & & -0.0347 & & 0.0011 & \\
\hline
\end{tabular}

The calculated coefficients of polynoms (5.1) are presented in Table 1.

Table 1. The polynomial coefficients at $\tau$ in a degree.

We see that the sets $\mathcal{R}_{j}$ in this case have more elements:

$$
\mathcal{R}_{1,2}=\{-6,-3,3,6\}, \mathcal{R}_{3,4}=\{-4,-2,-1,1,2,4\} .
$$

This means that not only the transverse oscillations induced the longitudinal ones as in example 1, but also the longitudinal oscillations induced a resonance (not only amplitude) effect on the transverse ones. This effect caused the first harmonic - the basic tone - of the transverse oscillations.

The constructed approximations of the Riemann invariants allow to write down the approximations of the required functions $u, v$, i.e. wave profile approximations:

$$
\begin{aligned}
& u(x, t ; \varepsilon) \approx \frac{1}{2 a} \int\left(R_{2}(\varepsilon t, x+a t)-R_{1}(\varepsilon t, x+a t)\right) d x \\
& =\left(1.3333+0.0394(\varepsilon t)^{2}-0.0033(\varepsilon t)^{4}\right) \sin 3(x+t)
\end{aligned}
$$




$$
\begin{aligned}
& -\left(0.6667+0.0197(\varepsilon t)^{2}-0.0016(\varepsilon t)^{4}\right) \cos 3(x+t) \\
& -\left(0.8333+0.0036(\varepsilon t)^{2}-0.0001(\varepsilon t)^{4}\right) \sin 3(x-t) \\
& +\left(0.1667+0.0007(\varepsilon t)^{2}\right) \cos 3(x-t) \\
& +\left(0.0208(\varepsilon t)-0.0005(\varepsilon t)^{3}\right) \sin 6(x+t) \\
& +\left(0.0069(\varepsilon t)-0.0002(\varepsilon t)^{3}\right) \cos 6(x+t) \\
& -\left(0.0556(\varepsilon t)+0.0068(\varepsilon t)^{3}+0.0007(\varepsilon t)^{5}\right) \sin 6(x-t) \\
& -\left(0.0139(\varepsilon t)+0.0017(\varepsilon t)^{3}+0.0002(\varepsilon t)^{5}\right) \cos 6(x-t) \text {, } \\
& v(x, t ; \varepsilon) \approx \frac{1}{2 b} \int\left(R_{4}(\varepsilon t, x+b t)-R_{3}(\varepsilon t, x+b t)\right) d x \\
& =\left(0.0208(\varepsilon t)-0,0002(\varepsilon t)^{3}\right) \sin (x+3 t) \\
& -\left(0.0764(\varepsilon t)-0.0009(\varepsilon t)^{3}\right) \cos (x+3 t) \\
& -\left(0.0278(\varepsilon t)-0,0009(\varepsilon t)^{3}\right) \sin (x-3 t) \\
& +\left(0.3611(\varepsilon t)-0.0123(\varepsilon t)^{3}-0.0005(\varepsilon t)^{5}\right) \cos (x-3 t) \\
& +\left(0.4167-0.0550(\varepsilon t)^{2}-0.0004(\varepsilon t)^{4}+0.0002(\varepsilon t)^{6}\right) \sin 2(x+3 t) \\
& +\left(0.2500+0.0330(\varepsilon t)^{2}+0.0002(\varepsilon t)^{4}-0.0001(\varepsilon t)^{6}\right) \cos 2(x+3 t) \\
& -\left(0.1667-0.0064(\varepsilon t)^{2}\right) \sin 2(x-3 t) \\
& +\left(0.0833-0.0032(\varepsilon t)^{2}\right) \cos 2(x-3 t) \\
& +\left(0.0417-0.0014(\varepsilon t)^{2}\right) \sin 4(x+3 t) \\
& -\left(0.04167-0.0014(\varepsilon t)^{2}\right) \cos 4(x+3 t) \\
& -\left(0.0417-0.0098(\varepsilon t)^{2}-0.0013(\varepsilon t)^{4}+0.0001(\varepsilon t)^{6}\right) \sin 4(x-3 t) \\
& +\left(0.0417-0.0098(\varepsilon t)^{2}+0.0013(\varepsilon t)^{4}-0.0001(\varepsilon t)^{6}\right) \cos 4(x-3 t) \text {. }
\end{aligned}
$$

The Figures 2-5 present the graphs of the functions $u$ and $v$ at different moments of the slow time $\tau=\varepsilon t$. Each graph contains three values of the fast time $t: t=10 \tau, t=100 \tau, t=1000 \tau$.

\section{Conclusions}

The system constructed by means of internal averaging along characteristics allows finding asymptotic solutions, elucidating their properties, determining the resonance-appearing conditions. 

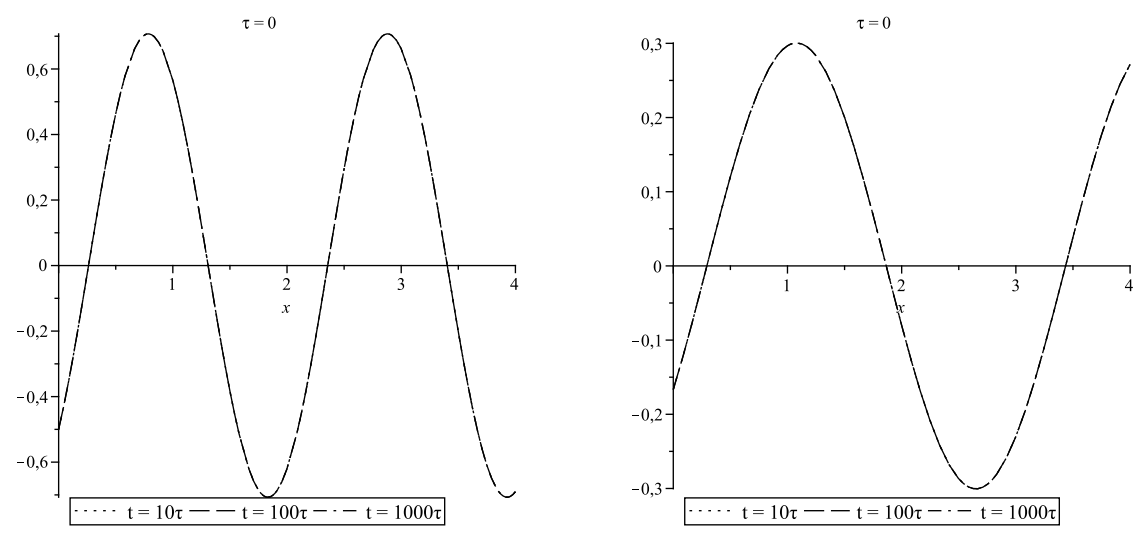

Figure 2. Profiles of waves $u, v$ at time moment $\tau=0$
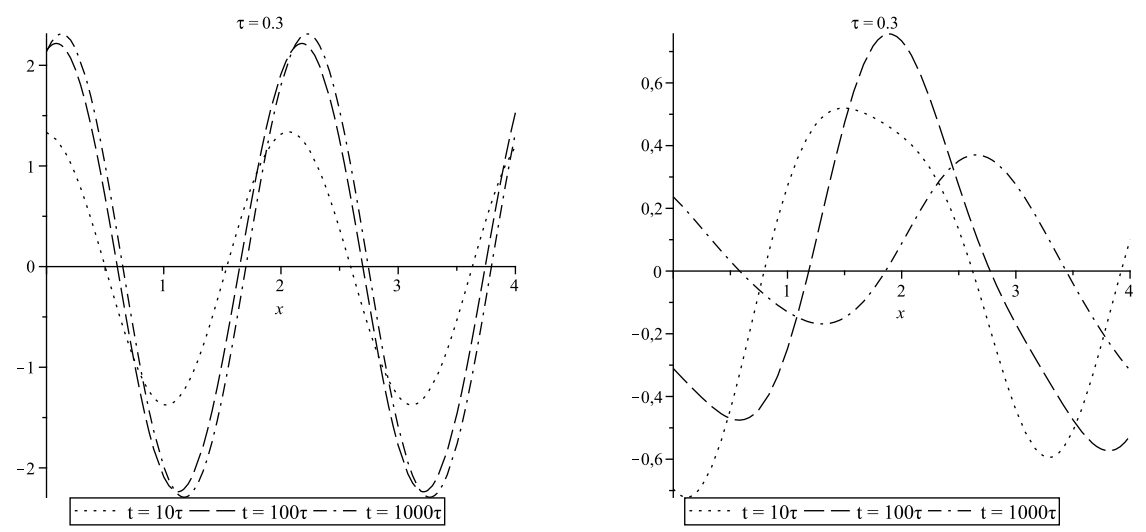

Figure 3. Profiles of waves $u, v$ at time moment $\tau=0.3$

The small-amplitude periodic oscillations in the Carrier system for a nonresonance case are described by independent waves travelling to different sides with the velocities $a=\sqrt{\frac{E}{\rho}}$ by transverse and $b=\sqrt{\frac{T}{\rho A}}$ by longitudinal waves.

In the resonance case, wave amplitudes depend on the slow time $\tau$, and the periodic functions describing the waves have the harmonics which have been absent at the initial moment of time. The resonant interaction of transverse and longitudinal waves may appear only if relations (4.2), (4.3) and (4.4) among the harmonics and the velocities $a, b$ are valid.

If at the initial moment of time the transverse oscillations contain no harmonics satisfying the relation (4.2), no resonance appears in the system. This means that the longitudinal oscillations alone cannot arouse the transversal ones, but the transverse oscillations may induce the longitudinal ones in the presence of (4.2).

In this case, the longitudinal oscillations, together with the transversal, ones may induce new harmonics of the transverse vibrations (overtones) of (4.2). 

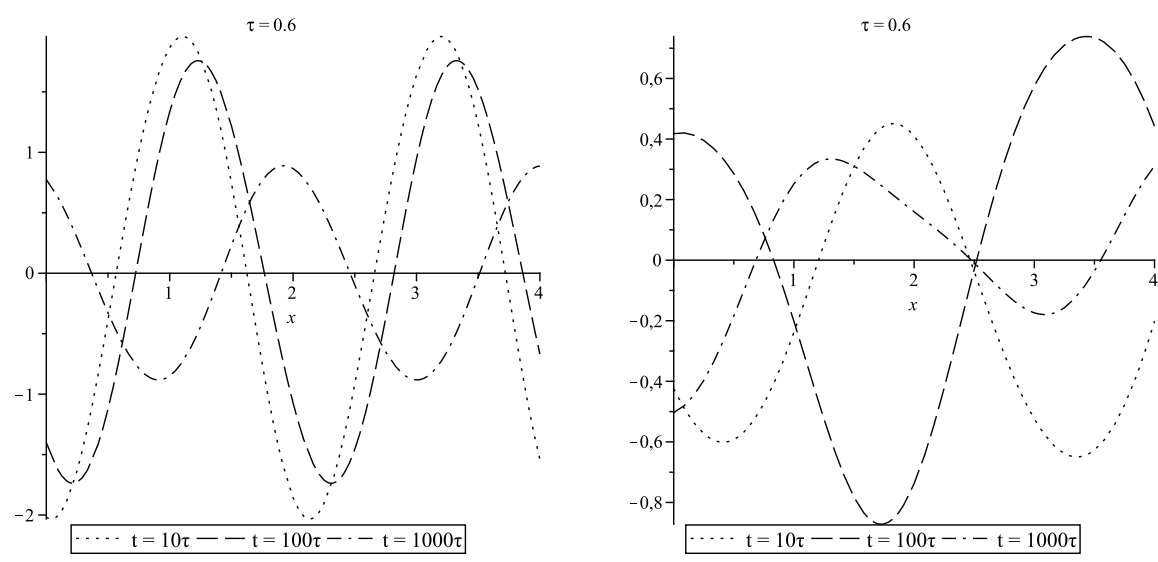

Figure 4. Profiles of waves $u, v$ at time moment $\tau=0.6$
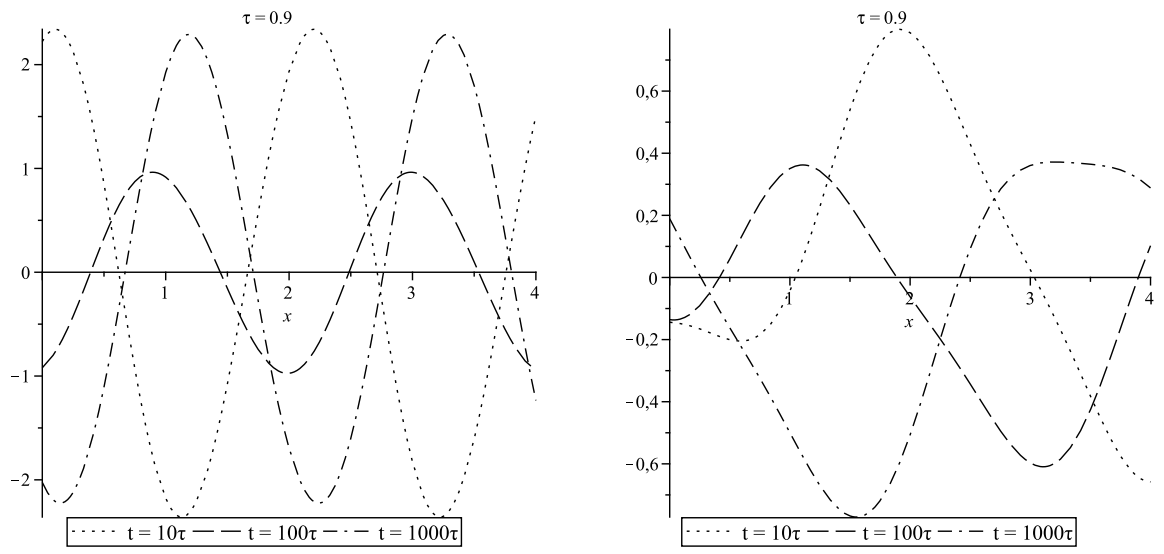

Figure 5. Profiles of waves $u, v$ at time moment $\tau=0.9$

Note that all conclusions are valid for the first asymptotic approximations (2.2). By presenting in the system (2.2) terms of the higher order of the small parameter $\varepsilon$, we may construct the second asymptotic approximation and analyze the harmonics which appear not only because of combinatoric resonances $(4.2),(4.3),(4.4)$ but also because of the system's nonlinearities. It is obvious that these effects will be of a higher $\varepsilon$ order, i. e. they will be weaker.

\section{References}

[1] W.F. Ames, S.Y. Lee and A.A. Vicario. Longitudinal wave propagation on a traveling threadline-II. International Journal of Non-Linear Mechanics, 5(3):413426, 1970. https://doi.org/10.1016/0020-7462(70)90004-1.

[2] W.F. Ames and A.A. Vicario. On the longitudinal wave propagation on a traveling threadline. Dev. Mech., 3(5):733-746, 1969. 
[3] R. Arora. Asymptotical solutions for a vibrationally relaxing gas. Mathematical Modelling and Analysis, 14(4):423-434, 2009. https://doi.org/10.3846/13926292.2009.14.423-434.

[4] G.K. Batchelor. An Introduction to Fluid Dynamics. Cambridge Mathematical Library. Cambridge University Press, 1967. https://doi.org/10.1017/CBO9780511800955.

[5] G.F. Carrier. On the non-linear vibration problem of the elastic string. Quarterly of applied mathematics, 3:157-165, 1945. https://doi.org/10.1090/qam/12351.

[6] G.F. Carrier. A note on the vibrating string. Quarterly of applied mathematics, 7:97-101, 1949. https://doi.org/10.1090/qam/28511.

[7] L.-Q. Chen. Analysis and control of transverse vibrations of axially moving strings. Applied Mechanics Reviews, 58(2):91-116, 2005. https://doi.org/10.1115/1.1849169.

[8] L.-Q. Chen and H. Ding. Two nonlinear models of a transversely vibrating string. Archive of Applied Mechanics, 78(5):321-328, 2008. https://doi.org/10.1007/s00419-007-0164-7.

[9] E.V. Ferapontov and K.R. Khusnutdinova. The Haantjes tensor and double waves for multi-dimensional systems of hydrodynamic type: a necessary condition for integrability. Proceedings of the Royal Society A, 462:1197-1219, 2006. https://doi.org/10.1098/rspa.2005.1627.

[10] E.V. Ferapontov and D.G. Marshall. Differential-geometric approach to the integrability of hydrodynamic chains: the Haantjes tensor. Mathematische Annalen, 339(1):61-99, 2007. https://doi.org/10.1007/s00208-007-0106-2.

[11] E.R. Gutierrez, P.L. Silva Dias and C. Raupp. Asymptotic approach for the nonlinear equatorial long wave interactions. Journal of Physics: Conference Series, 285(1):012020, 2011.

[12] V.V. Kozlov and S.D. Furta. Asymptotic Solutions of Strongly Nonlinear Systems of Differential Equations. Springer Monographs in Mathematics. SpringerVerlag, Berlin, Heidelberg, 2013.

[13] A. Krylovas. Justification of the method of internal averaging along characteristics of weakly linear systems. I. Lithuanian Mathematical Journal, 29(4):721$732,1989$.

[14] A. Krylovas. Justification of the method of internal averaging along characteristics of weakly nonlinear systems. II. Lithuanian Mathematical Journal, 30(1):3543, 1990. https://doi.org/10.1007/BF00966457.

[15] A. Krylovas and R. Čiegis. Asymptotic approximation of hyperbolic weakly nonlinear system. Journal Nonlinear Mathematical Physics, 8(4):458-470, 2001. https://doi.org/10.2991/jnmp.2001.8.4.2.

[16] A. Krylovas and R. Čiegis. A review of numerical asymptotic averaging for weakly nonlinear hyperbolic waves. Mathematical Modelling and Analysis, 9(3):209-222, 2004. https://doi.org/10.1080/13926292.2004.9637254.

[17] A. Krylovas, O. Lavcel-Budko and P. Miškinis. Asymptotic solution of the mathematical model of nonlinear oscillations of absolutely elastic inextensible weightless string. Nonlinear Analysis: Modelling and Control, 15(3):307-323, 2010.

[18] A. Krylovas and P. Miškinis. Nonlinear oscillations of the absolute elastic weightless string. Asymptotics construction. Lithuanian Mathematical Journal, spec. issue, 47:123-127, 2007. 
[19] A. Krylovas and P. Miškinis. Properties of averaging along the characteristics hyperbolic of the system operator. Lithuanian Mathematical Journal, spec. issue, 53(B):25-30, 2012.

[20] E. Kurihara and T. Yano. Nonlinear analysis of periodic modulation in resonances of cylindrical and spherical acoustic standing waves. Physics of Fluids, 18(11):117107, 2006. https://doi.org/10.1063/1.2393437.

[21] A.W. Leissa and A.M. Saad. Large amplitude vibrations of strings. Journal of Applied Mechanics, 61(2):296-301, 1994. https://doi.org/10.1115/1.2901444.

[22] P. Miškinis. Nonlinear and nonlocal integrable models (in Lithuanian). Technika, Vilnius, Lithuania, 2003.

[23] Y.A. Mitropolsky and Nguen Van Dao. Applied Asymptotic Methods in Nonlinear Oscillations. Solid Mechanics and Its Applications. Springer, Netherlands, 1997. https://doi.org/10.1007/978-94-015-8847-8.

[24] Y.A. Mitropolsky, G. Khoma and M. Gromyak. Asymptotic Methods for investigating Quasiwave Equations of Hyperbolic Type. Kluwer Academic Publishers, Dordrecht, 2006.

[25] A.S. Vasudeva Murthy. On the string equation of Narasimha. In Rajendra (ed.) et al. Bhatia(Ed.), Connected at infinity II. A selection of mathematics by Indians, pp. 58-84, New Delhi, 2012. Hindustan Book Agency. Texts and Readings in Mathematics 67.

[26] A. Nayfeh. Introduction to Perturbation Technigues. John Wiley \& Sons, Inc., New York, Chichester, Brisbabe, Toronto, 1981.

[27] D.E. Pelinovsky, G. Simpson and M.I. Weinstein. Polychromatic solytary waves in a periodic and nonlinear Maxwell system. SIAM Journal on Applied Dynamical Systems, 11(1):478-506, 2012. https://doi.org/10.1137/110837899.

[28] V.D. Sharma and G.K. Srinivasan. Wave interaction in a nonequilibrium gas flow. International Journal of Non-Linear Mechanics, 40(7):1031-1040, 2005. https://doi.org/10.1016/j.ijnonlinmec.2005.02.003.

[29] G. Simpson and M.I. Weinstein. Coherent structures and carries shocks in the nonlinear periodic Maxwell equation. Multiscale Modeling \& Simulation, 9(3):955-990, 2011. https://doi.org/10.1137/100810046.

[30] W.A. Strauss. Partial Differential Equations. An Introduction. Jon Wiley \& Sons, Ltd., 2008.

[31] T. Witelski and M. Bowen. Methods of Mathematical Modelling. Continuous Systems and Differential Equations. Springer Undergraduate Mathematics Series. Springer International Publishing, Switzerland, 2015. https://doi.org/10.1007/978-3-319-23042-9. 